\title{
ECONOMIA DA GESTÃO DE RESÍDUOS SÓLIDOS: O ESTADO DA ARTE EM UMA PERSPECTIVA INTERNACIONAL
}

\section{José Salvador da Motta Reis ${ }^{1}$, Fernanda De Oliveira Silva ${ }^{2}$, Maximilian Espuny ${ }^{1}$, Weslei Jardim Batista ${ }^{1}$ e Otávio José de Oliveira'.}

\author{
${ }^{1}$ Universidade Estadual Paulista /Faculdade de Engenharia de Guaratinguetá, Avenida Dr. Ariberto \\ Pereira da Cunha, 333, Bairro - 12244-000 - Guaratinguetá-SP, Brasil, jmottareis@gmail.com, \\ maximilian.espuny@unesp.br, wesleibatista.adm@gmail.com e otavio.oliveira@unesp.br. \\ ${ }^{2}$ Universidade Federal do ABC, Alameda da Universidade, s/nº, Anchieta - 09606-045 - São Bernardo \\ do Campo - SP, Brasil, fernanda.o@aluno.ufabc.edu.br.
}

\begin{abstract}
Resumo - O crescente aumento dos países europeus tem impactado de maneira significativa no volume de resíduos descartados. E, por outro lado, o desenvolvimento econômico chinês deu-se à luz de seus recursos naturais. Esta pesquisa pode ser classificada como aplicada, de natureza exploratória e abordagem quantitativa. Os métodos e procedimentos técnicos adotados foram, respectivamente, a pesquisa bibliográfica e a revisão de literatura. Percebeu-se um destaque por parte da China com o maior volume de produções com relação ao tema. O objetivo deste trabalho encontrava-se em identificar o estado de arte do tema voltado à economia da gestão de resíduos sólidos em uma perspectiva internacional, com a finalidade de destacar os principais países, periódicos, autores e palavras-chave referentes ao tema. Os objetivos de estudo foram alcançados, tendo em vista as principais descobertas referentes à bibliometria. Deste modo, pode-se auferir que o trabalho contribuiu par a analisar a evolução deste campo de pesquisa no âmbito acadêmico.
\end{abstract}

Palavras-chave: Estado da arte, blibliometria, gestão de resíduos, resíduos sólidos.

Área do Conhecimento: Engenharias.

\section{Introdução}

A relação entre a indústria e o meio ambiente é essencial para o incremento e a manutenção da atividade produtiva (LIEDER; RASHID, 2016). E pode ser notado que o desenvolvimento econômico acentuado por parte da China, nas últimas décadas, muito se deve aos seus recursos naturais (CHEN et al., 2012). Por outro lado, também, o fato de a Europa ter se tornado um continente rico, impacta de maneira significativa no volume de resíduos descartados causado pelo aumento de consumo por parte de sua população (MALINAUSKAITE et al., 2017).

Os custos que a disposição final e o sistema de coleta de resíduos sólidos podem alcançar percentuais de 0,1 a $0,4 \%$ do PIB de uma nação, e famílias de alguns países do mundo podem pagar o equivalente de 200 a 300 euros ao ano. Porém, além da perspectiva dos custos monetários, devese considerar principalmente os custos não monetários que, apesar de serem intangíveis, podem impactar de maneira irreversível o meio ambiente, como, por exemplo, a emissão de metais pesados causando dificuldades respiratórias à população (CHRISTENSEN, 2011).

Os custos que envolvem a gestão de resíduos sólidos devem ser providos pelo poder público ou pelo agente gerador dos resíduos. O custo do gerenciamento de resíduos residenciais pode estar embutido por impostos de propriedade (CHRISTENSEN, 2011).

Para uma melhor compreensão sobre os instrumentos econômicos na perspectiva da gestão de resíduos sólidos, buscou-se responder a seguinte questão de pesquisa: qual é o estado da arte voltado à economia da gestão de resíduos sólidos em uma perspectiva internacional? Para a obtenção de possíveis respostas, o objetivo deste artigo foi realizar um estudo bibliométrico, identificando os principais países, periódicos, autores e palavras-chave que compõe este campo do conhecimento. $O$ artigo encontra-se organizado nas seguintes seções: introdução, metodologia, resultados, discussão, conclusão, agradecimentos e referências. 


\section{Metodologia}

A pesquisa pode ser classificada como aplicada, de natureza exploratória e abordagem quantitativa. Como método e procedimentos técnicos foram adotados, respectivamente, a pesquisa bibliográfica e a revisão de literatura. Destaca-se que os dados foram coletados no mês de junho de 2019 na base de dados Scopus e que na busca foram utilizadas como descritores as palavras "Electronic Waste Economy", "Circular Economy", "Recycle Economics", "Informal Economy Waste" e "E-waste Economy", sendo encontrados na pesquisa (título) 263 resultados. Utilizou-se o recorte temporal de 2009 a 2019.

As informações contidas na pesquisa foram hierarquizadas pelo número de índice $\mathrm{H}$, dados estes que são projetados pela quantidade de artigos produzidos, com equivalência no número de citações por cada um dos artigos. Se um determinado autor possui três artigos produzidos, porém, dois artigos contivessem três citações e um artigo apresenta duas citações, logo, o suposto autor teria um índice H "2". Mas, se esse autor tivesse quatro artigos e todos eles dispusessem de quatro citações, o seu índice H seria "4". Para fazerem parte do ranking, os integrantes da pesquisa precisaram ter ao menos um Índice $\mathrm{H}$ de pelo menos 3.

Nos objetos de análise que obtiveram um empate em seu respectivo índice $\mathrm{H}$, obteve-se como critério de desempate o número de citações totais que o elemento analisado tivesse apresentado. Caso o empate tivesse sido permanente, o critério passaria a ser o número de publicações realizados. Para o aprofundamento da análise, utilizou-se também, a descrição dos últimos 10 anos de produção científica, assim como também os últimos 10 anos de citações obtidas.

Os dados foram tratados por meio dos softwares Microsoft Excel® e VOS Viewer e os resultados obtidos serão apresentados e discutidos na próxima seção.

\section{Resultados}

Buscou-se analisar neste estudo bibliométrico os três aspectos mais relevantes que contribuem para esta revisão teórica, sendo eles: autores, periódicos e países de maiores níveis de produtividade. Verificou-se que embora o foco da pesquisa estivesse em mapear os termos que associassem menções genéricas de economia mesclados com a gestão de resíduos sólidos, o que mais fora apontado nas pesquisas, em relação à economia, foram os termos "Economia Circular", sendo que apenas 15 artigos não apresentaram o termo específico, e obtiveram outras variações da palavra "economia", como por exemplo "economia de resíduos".

Os dados apresentados na Tabela 1 apontam os 10 autores mais citados na literatura sobre Economia de Resíduos, índice $\mathrm{H}$ do autor na área.

Tabela 1 - Autores mais citados entre 2009 e 2019.

\begin{tabular}{|c|c|c|c|c|c|c|c|c|c|c|c|c|c|c|c|c|c|c|c|c|c|c|c|c|}
\hline Autor & Índice H & $\begin{array}{c}\text { № de } \\
\text { documentos }\end{array}$ & $\begin{array}{c}\text { № de } \\
\text { citações }\end{array}$ & \multicolumn{10}{|c|}{$\begin{array}{c}\text { Documentos } \\
2009-2019 \\
\end{array}$} & \multicolumn{11}{|c|}{$\begin{array}{c}\text { Citações } \\
2009-2019 \\
\end{array}$} \\
\hline $\begin{array}{l}\text { Yong Geng } \\
\text { China }\end{array}$ & 8 & 9 & 684 & $\underline{1}$ & 0 & 0 & & 1 & 0 & $\underline{1}$ & 2 & 1 & 0 & 0 & $\stackrel{5}{-}$ & $\begin{array}{l}10 \\
-\end{array}$ & $\stackrel{13}{-}$ & $\begin{array}{l}17 \\
-\end{array}$ & $\begin{array}{l}29 \\
-\end{array}$ & $\begin{array}{c}50 \\
-\end{array}$ & $\begin{array}{l}97 \\
-\end{array}$ & 144 & 1991 & 120 \\
\hline $\begin{array}{l}\text { Phil Purnell } \\
\text { Reino Unido }\end{array}$ & 4 & 4 & 87 & 0 & 0 & 0 & 00 & 0 & 0 & 0 & 2 & $\underline{z}$ & 0 & 0 & 0 & 0 & 0 & 0 & 0 & 0 & 0 & $\frac{5}{-}$ & 40 & 42 \\
\hline $\begin{array}{l}\text { Costas A. Velis } \\
\text { Reino Unido }\end{array}$ & 4 & 4 & 87 & 0 & 0 & 0 & 00 & 0 & 0 & 0 & 2 & $\underline{2}$ & 0 & 0 & 0 & 0 & 0 & 0 & 0 & 0 & 0 & $\frac{5}{-}$ & 40 & 42 \\
\hline $\begin{array}{l}\text { Eleni lacovidou } \\
\text { Reino Unido }\end{array}$ & 4 & 4 & 87 & 0 & 0 & 0 & 00 & 0 & 0 & 0 & 2 & 2 & 0 & 0 & 0 & 0 & 0 & 0 & 0 & 0 & 0 & $\frac{5}{-}$ & 40 & 42 \\
\hline $\begin{array}{c}\text { Jhuma Sadhukhan } \\
\text { Reino Unido }\end{array}$ & 4 & 4 & 63 & 0 & 0 & 0 & 00 & 0 & 0 & 2 & 1 & 1 & 0 & 0 & 0 & 0 & 0 & 0 & 0 & 0 & $\begin{array}{l}5 \\
-\end{array}$ & 13 & 29 & 16 \\
\hline $\begin{array}{l}\text { Tsuyoshi Fujita } \\
\text { Japão }\end{array}$ & 3 & 3 & 240 & 1 & 0 & 0 & 11 & 10 & 0 & 0 & 0 & 0 & 0 & 0 & $\frac{5}{-}$ & $\begin{array}{c}10 \\
-\end{array}$ & $\frac{11}{-}$ & $\begin{array}{l}7 \\
-\end{array}$ & $\begin{array}{l}19 \\
=\end{array}$ & $\begin{array}{l}27 \\
=\end{array}$ & 43 & 42 & 54 & 22 \\
\hline $\begin{array}{l}\text { Liang Dong } \\
\text { Hong Kong }\end{array}$ & 3 & 3 & 99 & 0 & 0 & 0 & 01 & 0 & 0 & 1 & 1 & 0 & 0 & 0 & 0 & 0 & 0 & 0 & 8 & $\begin{array}{l}11 \\
\mathbf{m}\end{array}$ & 20 & 17 & 26 & 17 \\
\hline $\begin{array}{l}\text { Andrew Brown } \\
\text { Reino Unido }\end{array}$ & 3 & 3 & 77 & 0 & 0 & 0 & 00 & 0 & 0 & 0 & 2 & 1 & 0 & 0 & 0 & 0 & 0 & 0 & 0 & 0 & 0 & $\begin{array}{l}5 \\
-\end{array}$ & 35 & 37 \\
\hline
\end{tabular}

XXIII Encontro Latino Americano de Iniciação Científica, XIX Encontro Latino Americano de Pós-Graduação e 2 IX Encontro de Iniciação à Docência - Universidade do Vale do Paraíba. 


$\begin{array}{cccccccccccccccccccccccccccccc}\begin{array}{c}\text { Oliver Zwirner } \\ \text { Reino Unido }\end{array} & 3 & 3 & 77 & 0 & 0 & 0 & 0 & 0 & 0 & 0 & 0 & 2 & 1 & 0 & 0 & 0 & 0 & 0 & 0 & 0 & 0 & 0 & 5 & 35 & 37 \\ \begin{array}{c}\text { Joel Millward-Hopkins } \\ \text { Reino Unido }\end{array} & 3 & 3 & 77 & 0 & 0 & 0 & 0 & 0 & 0 & 0 & 0 & 2 & 1 & 0 & 0 & 0 & 0 & 0 & 0 & 0 & 0 & 0 & 5 & 35 & 37\end{array}$

Fonte: Próprios autores.

Conforme a Tabela 1, observa-se que as primeiras publicações foram realizadas por Geng e por Fujita, a partir de 2009. Geng apresenta um volume de produções bastante expressiva, obtendo uma média de quase um artigo por ano, conforme o período analisado. Fujita, apresenta três produções, porém, com uma média de 80 citações por artigo, superior inclusive a média de citações obtida por Geng (76 citações por artigo).

Observa-se que existem dois grupos com três autores (Purmell, Velis e lacovidou; Browm, Zwimer e Millward-Hopkins), que realizaram respectivamente quatro e três artigos, obtendo o volume de 87 e 77 citações totais. Ambos os trios pertencem a instituições do Reino Unido, país este que predomina destacando sete de 10 autores que predomina na lista destacada acima.

Verifica-se que o volume de citações, de maneira geral, obtém um crescimento constante, alcançando apontamentos superiores aos apresentados nos respectivos anos anteriores, com algumas exceções na linha histórica de Fujita.

Os dados apresentados na Tabela 2 apontam os 10 periódicos mais citados na literatura sobre Economia de Resíduos, índice $\mathrm{H}$ do periódico na área.

Tabela 2 - Periódicos mais citados entre 2009 e 2019.

\begin{tabular}{|c|c|c|c|c|c|c|c|c|c|c|c|c|c|c|c|c|c|c|c|c|c|c|c|c|}
\hline Periódico & Índice $\mathrm{H}$ & $\begin{array}{c}\text { № de } \\
\text { documentos }\end{array}$ & $\begin{array}{c}\text { № de } \\
\text { citações }\end{array}$ & \multicolumn{10}{|c|}{$\begin{array}{c}\text { Documentos } \\
2009 \text { - } 2019 \\
\end{array}$} & \multicolumn{11}{|c|}{$\begin{array}{c}\text { Citações } \\
2009-2019 \\
\end{array}$} \\
\hline $\begin{array}{l}\text { Journal of Cleaner } \\
\text { Production }\end{array}$ & 16 & 46 & 2042 & 0 & 0 & 0 & $\begin{array}{l}1 \\
-\end{array}$ & $\begin{array}{l}2 \\
-\end{array}$ & 0 & $\begin{array}{l}3 \\
-\end{array}$ & $\begin{array}{l}6 \\
.\end{array}$ & 8 & $10^{16}$ & 0 & 0 & 0 & 2 & 10 & $\begin{array}{l}26 \\
-\end{array}$ & $\begin{array}{l}42 \\
-\end{array}$ & 152 & $\begin{array}{c}379 \\
\square\end{array}$ & & 9712 \\
\hline $\begin{array}{l}\text { Resources Conservation } \\
\text { And Recycling's }\end{array}$ & 10 & 26 & 273 & $\begin{array}{l}1 \\
-\end{array}$ & 0 & 0 & 0 & 0 & 0 & 1 & $\begin{array}{l}3 \\
-\end{array}$ & $\begin{array}{l}4 \\
-\end{array}$ & $\begin{array}{l}125 \\
\square \quad-\end{array}$ & 0 & 3 & $\begin{array}{l}4 \\
-\end{array}$ & $\begin{array}{l}7 \\
-\end{array}$ & $\begin{array}{l}5 \\
-\end{array}$ & $\begin{array}{l}8 \\
-\end{array}$ & $\begin{array}{l}7 \\
-\end{array}$ & $\begin{array}{l}11 \\
-\end{array}$ & $\begin{array}{l}25 \\
=\end{array}$ & 85 & 118 \\
\hline $\begin{array}{l}\text { Journal of Industrial } \\
\text { Ecology }\end{array}$ & 7 & 9 & 244 & 0 & 0 & 0 & 2 & 0 & 0 & 0 & 0 & 5 & 02 & 0 & 0 & 0 & $\stackrel{5}{-}$ & $\frac{4}{-}$ & $\begin{array}{l}13 \\
-\end{array}$ & $\begin{array}{c}18 \\
-\end{array}$ & $\begin{array}{l}16 \\
-\end{array}$ & $\begin{array}{l}30 \\
=\end{array}$ & 78 & 80 \\
\hline Waste Management & 7 & 20 & 225 & $\begin{array}{l}1 \\
-\end{array}$ & 0 & 0 & $\begin{array}{l}1 \\
-\end{array}$ & $\begin{array}{l}1 \\
-\end{array}$ & 0 & 0 & 0 & 3 & & 0 & $\frac{5}{-}$ & $\begin{array}{l}10 \\
-\end{array}$ & $\begin{array}{l}8 \\
-\end{array}$ & $\begin{array}{l}10 \\
-\end{array}$ & $\begin{array}{l}6 \\
-\end{array}$ & $\begin{array}{l}15 \\
-\end{array}$ & $\begin{array}{l}19 \\
=\end{array}$ & 35 & 64 & $\begin{array}{r}53 \\
-1\end{array}$ \\
\hline Bioresource Technology & 5 & 6 & 167 & 0 & 0 & 0 & 0 & 0 & 0 & 0 & 2 & 2 & 11 & 0 & 0 & 0 & 0 & 0 & 0 & 0 & 7 & $\begin{array}{l}35 \\
\text { ! }\end{array}$ & 72 & 53 \\
\hline $\begin{array}{c}\text { Waste Management And } \\
\text { Research }\end{array}$ & 4 & 5 & 81 & 0 & 0 & 0 & 0 & 0 & 0 & 0 & 0 & $\frac{2}{-}$ & $7^{11}$ & 0 & 0 & 0 & 0 & 0 & 0 & 0 & 0 & 1 & & 21 \\
\hline $\begin{array}{c}\text { Journal of Industrial } \\
\text { Ecology }\end{array}$ & 4 & 7 & 62 & 0 & 0 & 0 & 0 & 0 & 0 & 0 & 0 & 1 & 33 & 0 & 0 & 0 & 0 & 0 & 0 & 0 & 0 & 2 & 24 & 36 \\
\hline $\begin{array}{l}\text { Science of The Total } \\
\text { Environment }\end{array}$ & 4 & 12 & 42 & 0 & 0 & 0 & 0 & 0 & 0 & 0 & 5 & 2 & 32 & 0 & 0 & 0 & 0 & 0 & 0 & 0 & 0 & $\begin{array}{l}7 \\
\text { [ }\end{array}$ & 17 & 18 \\
\hline Bioresource Technology & 3 & 5 & 84 & 0 & 0 & 0 & 0 & 0 & 1 & 0 & 1 & 0 & 03 & 0 & 0 & 0 & 0 & 0 & 1 & $\begin{array}{l}7 \\
-\end{array}$ & 9 & $\begin{array}{l}17 \\
\mathbf{⿴ 囗 十}\end{array}$ & 20 & 30 \\
\hline $\begin{array}{c}\text { Journal of Environmental } \\
\text { Management }\end{array}$ & 3 & 20 & 35 & 0 & 0 & 0 & 0 & 0 & 0 & 0 & 0 & 2 & $7^{11}$ & 0 & 0 & 0 & 0 & 0 & 0 & 0 & 0 & 1 & & $\begin{array}{l}21 \\
\square\end{array}$ \\
\hline
\end{tabular}

Fonte: Próprios autores.

Observa-se uma predominância bastante considerável do Journal of Clenaer Production alcançando 46 publicações nos últimos oito anos. Destaca-se também, os periódicos Resources Conservation and Recycling (26 publicações), Waste Management e Journal of Environmental Management (20 publicações cada uma delas).

Os periódicos Resources Conservation and Recycling e Waste Management foram os primeiros a apresentarem uma publicação do tema, ambos em 2009. Verifica-se que todos os periódicos mencionados realizaram publicações no ano de 2019. 
Os dados apresentados na Tabela 3 apontam os 10 países mais citados na literatura sobre Economia de Resíduos, índice H do país na área.

Tabela 3 - Países mais citados entre 2009 e 2019.

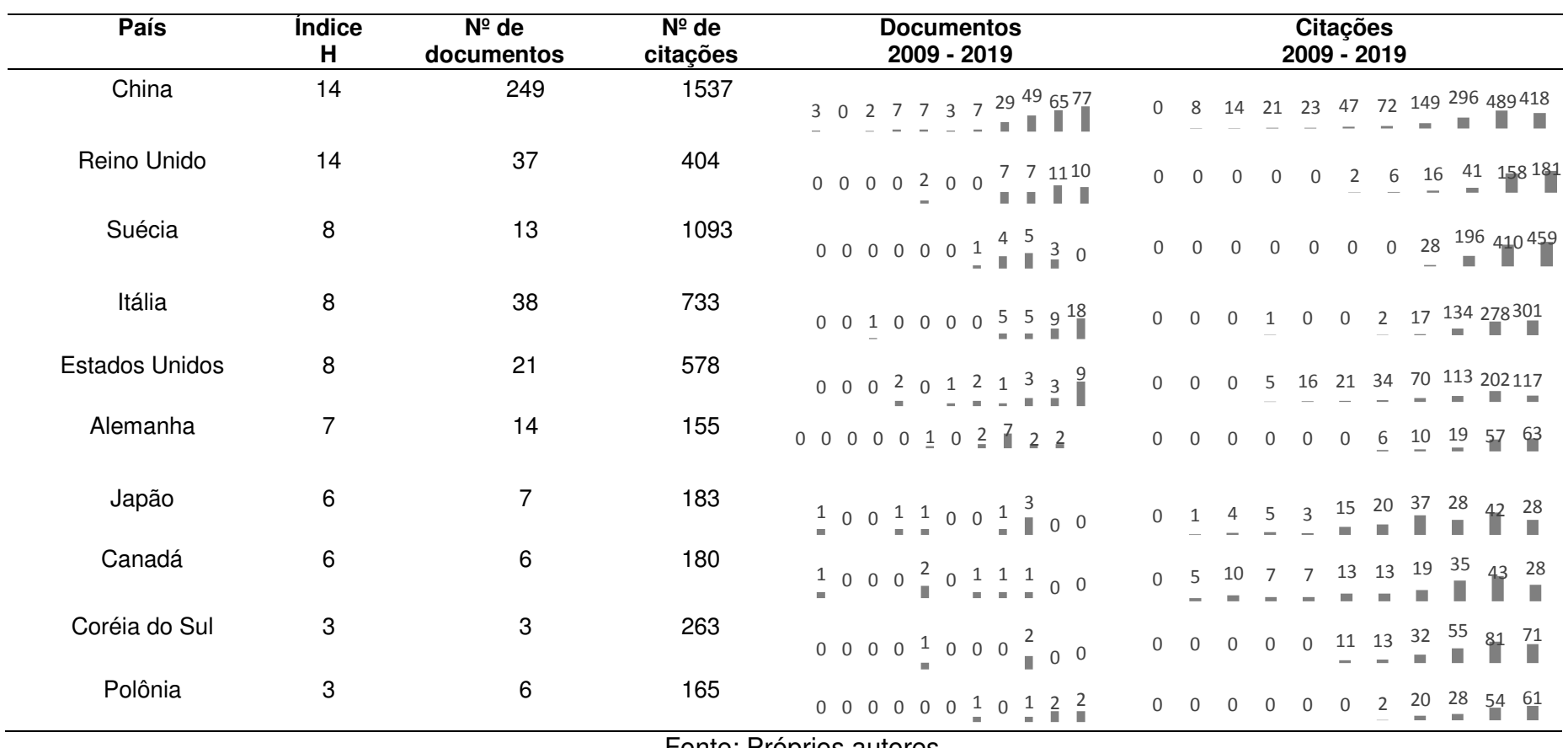

Fonte: Próprios autores.

Verifica-se que o índice $\mathrm{H}$ da China e do Reino Unido encontram-se empatados, com ambos apresentando, neste quesito, o valor de 14. Mas, chama a atenção o fato de a China concentrar 249 publicações, enquanto no Reino Unido apresenta apenas 37 publicações. Contudo, pelo fato de ambos os índices serem equivalentes, apesar de uma produção chinesa muito mais significativa, observa-se que o material produzido pelos britânicos é de uma contribuição acadêmica mais relevante.

A Suécia, que apresenta a terceira colocação em termos de relevância acadêmica, obtém um índice H 8, apesar de ter emplacado 13 artigos. A média de citações apresentada pela Suécia é em torno de 86 enquanto a China apresenta uma média de apenas seis citações por artigos. A Coréia do Sul apesar de apresentar apenas três artigos, possui uma média bastante relevante de 87 citações. Em 2019, a Suécia, o Japão, o Canadá e a Coréia do Sul não apresentaram nenhuma produção voltada a economia associada com resíduos sólidos.

Percebe-se que o volume total de citações vem aumentando ano a ano e embora nos gráficos sejam apresentados uma relativa diminuição de apontamentos em 2019, vale lembrar que as informações obtidas são referentes há um pouco mais da metade do ano ou seja, o potencial de crescimento é bastante considerável.

Entre outros resultados relevantes obtidos, verificou-se que as palavras-chave mais apontadas no estudo foram reciclagem (97 apontamentos), gestão de resíduos municipal (53 apontamentos), sustentabilidade (32 apontamentos), avaliação de ciclo de vida (24 apontamentos) e indústria ecológica (20 apontamentos), desconsiderando os termos que foram utilizados para captura das informações dessa pesquisa.

A área que mais apareceu estudos relacionados com esta pesquisa foi a de ciências ambientais (189 artigos), seguido por energia (83), engenharia (60); gestão, negócios e contabilidade (55) e ciências sociais (47).

As universidades que mais apresentaram estudos relacionados ao tema foram a Chinese Academy of Sciences (9 artigos) e a Danmarks Tekniske Universitet (8 artigos). Em relação aos fundos de financiamento, a Chinese Academy of Sciences (13 artigos) também desponta como a mais bem colocada, seguida por Natural Environment Researche Council (8 artigos). Apresentam-se 
também, na lista de fundos, três instituições brasileiras, sendo o CNPQ como fomentadora de seis estudos abrangendo o tema em voga.

\section{Discussão}

A gestão de resíduos sólidos passou a ser uma variável de vital importância dentro do universo da economia. Essa percepção passou a ser mais sensível devido à escassez de recursos materiais e aos impactos financeiros causados pelos resíduos. Com este cenário, os gestores públicos e os empresários não podem mais subestimar o enorme volume de estrago que uma gestão de resíduos ineficiente acarreta para as diversas partes interessadas, principalmente nas cidades, conforme Malinauskaite (2017).

Verifica-se que os países com maior interesse nessa área do conhecimento são os europeus ou os pertencentes das maiores economias, como a China, o Japão, Coréia do Sul e ainda os Estados Unidos. Em relação aos autores principais, há uma concentração de autores entre o Reino Unido e os países asiáticos.

A concentração da grande quantidade de estudos promovidos pela China, pode-se dar por conta das necessidades em gerir diversos aspectos que envolvam a sustentabilidade, devido a exploração de recursos que visem garantir crescimento econômico, conforme Chen (2012). A tendência de estudos voltado a economia de resíduos, muito presente por diversos países europeus pode ser por conta dos custos que possam impactar na condição do ecossistema do planeta e na saúde humana, conforme Christensen (2011).

\section{Conclusão}

Percebe-se nos últimos anos que o consumo desenfreado por parte da população e o uso intensivo dos recursos naturais têm apresentado como consequência o aumento do descarte de resíduos sólidos de maneira inapropriada no meio ambiente. Acarretando, deste modo, em consequências irreversíveis a esse. Devendo assim, ser gerida de modo eficiente pelo poder público e por seus agentes geradores.

O objetivo deste trabalho encontrava-se em identificar o estado da arte do tema voltado à economia da gestão de resíduos sólidos em uma perspectiva internacional, a fim de destacar os principais países, periódicos, autores e palavras-chave referentes ao tema. Os objetivos de estudo foram alcançados, tendo em vista as principais descobertas referentes à bibliometria. Deste modo, pode-se auferir que o trabalho contribuiu para analisar a evolução deste campo de pesquisa no âmbito acadêmico.

Observou-se que o volume de citações referente ao que foi abordado apresentou expressivo crescimento no período em análise. Com destaque para o autor Fujita, com a maior média de citações por artigo no período, e ao periódico Journal of Cleaner Production por apresentar o maior volume de citações entre 2009 e 2019.

Devendo-se ao aumento da preocupação chinesa em proporcionar um crescimento econômico sustentável ao país, percebeu-se que o país apresentou o maior número de publicações (249), concomitante ao maior volume de citações (1537). Sendo esse, seguido pelo Reino Unido com o índice $\mathrm{H}$ equivalente, todavia encontra-se em terceiro lugar referente ao volume de produções (37). Evidenciando, assim, que os trabalhos publicados pelos britânicos apresentam contribuição acadêmica de maior relevância se comparado as produções chinesas.

Percebe-se, também, um destaque às áreas de ciências ambientais e energia com, respectivamente, 189 e 83 artigos cada. A universidade Chinese Academy of Sciences, localizada na China, destacou-se sobre as demais com nove estudos relacionados ao tema

Verificou-se entre os elementos analisados, que se trata de um tema bastante recente e que passou a ter um volume de citações relevantes apenas a partir de 2013 e 2014. Isso pode ser um indicativo que muitas propostas de estudos podem ser elaboradas para os próximos anos, tanto para a comunidade acadêmica, como para as indústrias e principalmente aos gestores dedicados aos resíduos.

\section{Agradecimentos}

XXIII Encontro Latino Americano de Iniciação Científica, XIX Encontro Latino Americano de Pós-Graduação e 5 IX Encontro de Iniciação à Docência - Universidade do Vale do Paraíba. 
Este trabalho foi viabilizado com o apoio do CNPq PQ Proc. 312894/2017-1.

\section{Referências}

CHEN, X. et al. The Impact of Scale, Recycling Boundary, and Type of Waste on Symbiosis and Recycling. Journal of Industrial Ecology, v. 16, n. 1, p. 129-141, fev. 2012.

CHRISTENSEN, T. H. Solid Waste Technology \& Management. 1. ed. Chichester, UK: Wiley, 2011.

LIEDER, M.; RASHID, A. Towards circular economy implementation: a comprehensive review in context of manufacturing industry. Journal of Cleaner Production, v. 115, p. 36-51, mar. 2016.

MALINAUSKAITE, J. et al. Municipal solid waste management and waste-to-energy in the context of a circular economy and energy recycling in Europe. Energy, v. 141, p. 2013-2044, dez. 2017. 